\title{
Swapping of Gaussian Einstein-Podolsky-Rosen steering
}

\author{
Meihong Wang ${ }^{1}$, Zhongzhong $\mathrm{Qin}^{1,2}$ and Xiaolong $\mathrm{Su}^{1,2}$. \\ ${ }^{1}$ State Key Laboratory of Quantum Optics and Quantum Optics Devices, \\ Institute of Opto-Electronics, Shanxi University, \\ Taiyuan, 030006, People's Republic of China \\ ${ }^{2}$ Collaborative Innovation Center of Extreme Optics, \\ Shanxi University, Taiyuan 030006, China
}

\begin{abstract}
Einstein-Podolsky-Rosen (EPR) steering is a quantum mechanical phenomenon that allows one party to steer the state of a distant party by exploiting their shared entanglement. It has potential applications in secure quantum communication. In this paper, we present two swapping schemes of Gaussian EPR steering, single-channel and dual-channel schemes, by the technique of entanglement swapping. Two space-separated independent EPR steering states without a direct interaction present EPR steering after deterministic swapping. By comparing the EPR steering of the singlechannel and dual-channel schemes, we show that the transmission distance of the single-channel scheme is much longer than that of the symmetric dual-channel scheme. Different from entanglement swapping, one-way EPR steering is presented after swapping over lossy channels. The most interesting thing is that the change of the EPR steering direction is observed in the dual-channel scheme. We also show that excess noise in a quantum channel will shorten the transmission distance of the swapping, even leading to the sudden death of EPR steering. The presented schemes provide a technical reference for remote quantum communications with EPR steering.
\end{abstract}

\section{INTRODUCTION}

Einstein-Podolsky-Rosen (EPR) steering was first noted by Einstein, Podolsky, and Rosen in their famous 1935 paper [1]. They argued the completeness of quantum mechanics by calling this phenomenon "spooky action at a distance." In his response to the EPR paper, Schrödinger originally introduced the concept of EPR steering [2, 3]. Suppose Alice and Bob share an EPR entangled state and they are separated in space. EPR steering means that one party, say, Alice, can "steer" the state in Bob's station by performing a measurement on her state at a distance, i.e., if Alice makes a measurement on her state, the state in Bob's station will change instantaneously. In the hierarchy of quantum correlations, EPR steering represents a weaker form of quantum nonlocality and stands between Bell nonlocality [4] and EPR entanglement [5]. Concretely, the violation of Bell inequality implies EPR steering in both directions, and EPR steering of any direction implies that the quantum state is entangled [ [6].

EPR steering has recently attracted increasing interest in the quantum optics and quantum information communities [6-8]. EPR steering can be regarded as verifiable entanglement distribution by an untrusted party, while entangled states need both parties to trust each other, and Bell nonlocality is valid assuming that they distrust each other [7]. In the field of quantum information processing, EPR steering has potential applications in onesided device-independent quantum key distribution [9], channel discrimination [10], and teleamplification [11].

*Electronic address: suxl@sxu.edu.cn
The inherent asymmetric feature is the unique property of EPR steering that differs from both entanglement and Bell nonlocality. There are situations when Alice can steer Bob's state but Bob can not steer Alice's state, or vice versa, which are referred to as one-way EPR steering [6]. The demonstration of one-way EPR steering is of foundational significance in testing the basic laws in quantum mechanics and has potential applications in asymmetric quantum information processing. Gaussian one-way EPR steering has been demonstrated with a twomode squeezed state 12] and a multipartite EPR steering system [13], with their measurements restricted to Gaussian measurements. Other measurement methods used to show the property of one-way EPR steering have been theoretically constructed, including general projective measurements [14], arbitrary finite-setting positiveoperator-valued measures (POVMs) 8], infinite-setting POVMs [15], and infinite number of arbitrary projective measurements [16]. Very recently, genuine one-way EPR steering was experimentally demonstrated by two groups independently [17, 18], based on proposals in Refs. 16] and [14], respectively.

In a quantum network, the remote transfer of a quantum state is an important step in quantum communication. Entanglement swapping [19 23], which makes two independent quantum entangled states without a direct interaction become entangled, is an important technique in building a quantum information network 24]. In fact, it represents the quantum teleportation of an entangled state 22]. Entanglement swapping has been experimentally demonstrated in both discrete and continuousvariable regions [25, 26]. Recently, entanglement swapping between discrete and continuous-variable systems has been demonstrated [27]. Entanglement swapping among three two-photon EPR entangled states has been 
used to generate a Greenberger-Horne-Zeilinger state [28]. The technique of entanglement swapping has been applied to complete the remote transfer of Gaussian quantum discord [29]. Very recently, quantum entanglement swapping between two multipartite entangled states has been demonstrated experimentally [30], which shows the feasibility of connecting two multipartite entangled states by entanglement swapping.

In this paper, we apply the technique of entanglement swapping to realize the deterministic swapping of Gaussian EPR steering. We show that the steering property exists between two independent states without a direct interaction after entanglement swapping. Specifically, two kinds of swapping schemes are compared, which are called the single-channel scheme and dual-channel scheme, respectively. We theoretically analyze the swapping of EPR steering in a realistic environment, where the optical modes are transmitted in one or two lossy and noisy channels. The dependence of EPR steering on transmission distances and excess noise in quantum channels is presented. The results show that the transmission distance of a single-channel scheme is much longer than that of a symmetric dual-channel scheme. The most interesting result is that the change of the EPR steering direction is observed in the dual-channel scheme, which is a phenomenon related to the asymmetric property of the EPR state. The effect of excess noise in a quantum channel on output EPR steering is also analyzed. We show that excess noise can shorten the transmission distance of the swapping scheme and lead to the sudden death of EPR steering. The presented results provide a realistic reference to construct a quantum communication network with EPR steering.

\section{EPR STEERING SWAPPING SCHEMES}

Figure 1 shows the schematic of EPR steering swapping. Alice and Bob own two independent EPR entangled states $(\hat{A}, \hat{B})$ and $(\hat{C}, \hat{D})$, which have the property of EPR steering, respectively. There is no direct interaction between these two space-separated EPR states. In order to establish EPR steering between them, we apply the swapping scheme to them. We consider two kinds of swapping schemes, i.e., a single-channel scheme and dual-channel scheme, where the state is transmitted over a single quantum channel and two quantum channels, respectively.

In the single-channel scheme, as shown in Fig. 1(a), Alice sends mode $\hat{B}$ of her EPR state to Bob through a quantum channel. Bob performs a joint measurement on the received optical mode $\hat{B}$ and one of the EPR mode $\hat{C}$ hold by himself. The joint measurement is performed by coupling them on a 1:1 beam splitter and measuring the amplitude and phase quadratures of the output modes $\hat{E}$ and $\hat{F}$ by two homodyne detectors (HDs), respectively. The measurement results are fed forward to mode $\hat{D}$ by (a)

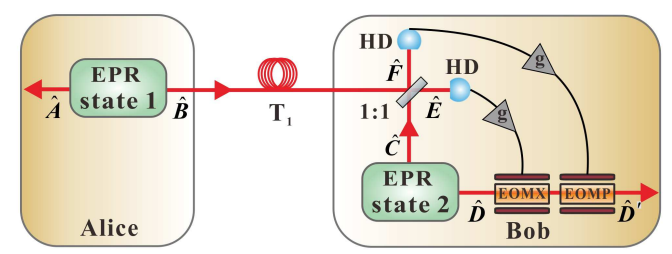

(b)

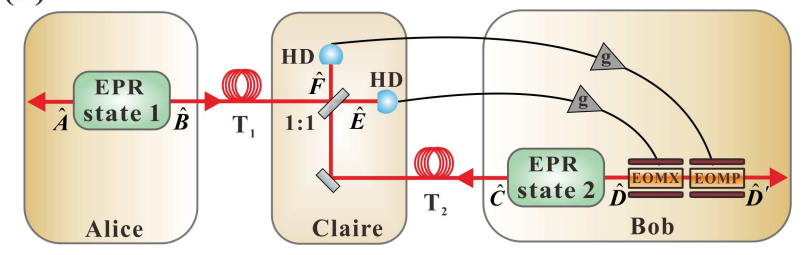

FIG. 1: The schematic of two swapping schemes of EPR steering. Alice and Bob own EPR entangled state $(\hat{A}, \hat{B})$ and $(\hat{C}, \hat{D})$, respectively. (a) Single-channel scheme. A joint measurement is performed at Bob's station and the measurement results are fed forward to mode $\hat{D}$. (b) Dual-channel scheme. A joint measurement is performed at Claire's station. HD: homodyne detector; EOMX and EOMP: amplitude and phase electro-optical modulators; $T_{1}, T_{2}$ : transmission efficiencies of the quantum channels.

classical channels. Bob performs phase-space displacement on quantum mode $\hat{D}$ with amplitude and phase modulators, respectively. In the dual-channel scheme, as shown in Fig. 1(b), Alice and Bob send one of each state $(\hat{B}$ and $\hat{C})$ to the middle station owned by Claire through two quantum channels, respectively. Claire performs a joint measurement on the received optical modes $\hat{B}$ and $\hat{C}$. The measurement results are fed forward to mode $\hat{D}$ through classical channels. Finally, EPR steering between $\hat{A}$ and $\hat{D}^{\prime}$ is verified.

In the dual-channel scheme, when the transmission distances of quantum channels $T_{1}$ and $T_{2}$ are different, it corresponds to an asymmetric swapping scheme. For the asymmetric swapping scheme, the middle station is placed near Alice's or Bob's station. The single-channel scheme is a special case of the asymmetric swapping scheme. When the transmission distances of quantum channels $T_{1}$ and $T_{2}$ are the same, it corresponds to a symmetric dual-channel scheme. In this case, the distances from the middle station to Alice's and Bob's station are the same.

The amplitude and phase quadratures of an optical mode $\hat{o}$ are defined as $\hat{x}_{o}=\hat{o}+\hat{o}^{\dagger}$ and $\hat{p}_{o}=\left(\hat{o}-\hat{o}^{\dagger}\right) / i$, respectively. Under this notation, the variances of the amplitude and phase quadratures for a vacuum state are $V\left(\hat{x}_{0}\right)=V\left(\hat{p}_{0}\right)=1$, where the subscript 0 represents the vacuum state. A Gaussian EPR entangled state with a variance $V=\cosh 2 r$, where $r \in[0, \infty)$ is the squeezing 
parameter, can be described by its covariance matrix

$$
\sigma_{A B}=\left(\begin{array}{cc}
V \mathbf{I} & \sqrt{V^{2}-1} \mathbf{Z} \\
\sqrt{V^{2}-1} \mathbf{Z} & V \mathbf{I}
\end{array}\right),
$$

with the matrix element $\sigma_{i j}=\left\langle\hat{\xi}_{i} \hat{\xi}_{j}+\hat{\xi}_{j} \hat{\xi}_{i}\right\rangle / 2-\left\langle\hat{\xi}_{i}\right\rangle\left\langle\hat{\xi}_{j}\right\rangle$, where $\hat{\xi} \equiv\left(\hat{x}_{A}, \hat{p}_{A}, \hat{x}_{B}, \hat{p}_{B}\right)$ is the vector of the field quadratures, and $\mathbf{I}$ and $\mathbf{Z}$ are the Pauli matrices

$$
\mathbf{I}=\left(\begin{array}{ll}
1 & 0 \\
0 & 1
\end{array}\right), \quad \mathbf{Z}=\left(\begin{array}{cc}
1 & 0 \\
0 & -1
\end{array}\right),
$$

respectively.

It has been shown that the steerability of a two-mode Gaussian state can be easily quantified by [31]

$$
\mathcal{G}^{A \rightarrow B}\left(\sigma_{A B}\right)=\max \left\{0, \frac{1}{2} \ln \frac{\operatorname{det} \sigma_{A}}{\operatorname{det} \sigma_{A B}}\right\},
$$

where $\sigma_{A}$ and $\sigma_{A B}$ are the covariance matrices corresponding to Alice's state and the two-mode Gaussian state, respectively. $\mathcal{G}^{A \rightarrow B}\left(\sigma_{A B}\right)>0$ represents that Alice has the ability to steer Bob's state. Similarly, we have

$$
\mathcal{G}^{B \rightarrow A}\left(\sigma_{A B}\right)=\max \left\{0, \frac{1}{2} \ln \frac{\operatorname{det} \sigma_{B}}{\operatorname{det} \sigma_{A B}}\right\},
$$

which represents Bob's ability to steer Alice's state, where $\sigma_{B}$ is the covariance matrix of Bob's state.

In the ideal case, i.e., with unit transmission efficiency and detection efficiency, the output modes from the $1: 1$ beam splitter are $\hat{E}=(\hat{B}-\hat{C}) / \sqrt{2}$ and $\hat{F}=(\hat{B}+\hat{C}) / \sqrt{2}$, respectively. The amplitude and phase quadratures of the optical modes $\hat{E}$ and $\hat{F}$ are measured by two homodyne detectors, respectively. The measurement results for the optical modes $\hat{E}$ and $\hat{F}$ are represented by $\hat{\imath}_{E}=\left(\hat{x}_{B}-\hat{x}_{C}\right) / \sqrt{2}$ and $\hat{\imath}_{F}=\left(\hat{p}_{B}+\hat{p}_{C}\right) / \sqrt{2}$, respectively. The measured results are fed forward to optical mode $\hat{D}$ through the classical channels, respectively. The output beam is

$$
\hat{D}^{\prime}=\hat{D}+\sqrt{2} g \hat{\imath}_{E}+i \sqrt{2} g \hat{\imath}_{F},
$$

where $g$ describes the amplitude and phase gain factor in the classical channels, and here we have assumed that the gains in the two classical channels are equal.

The covariance matrix of the output states $\hat{A}$ and $\hat{D}^{\prime}$ is given by

$$
\sigma_{\mathrm{out}}=\left(\begin{array}{cc}
A \mathbf{I} & C \mathbf{Z} \\
C \mathbf{Z} & B \mathbf{I}
\end{array}\right)
$$

where $A=V, B=\left(1+2 g^{2}\right) V-2 g \sqrt{V^{2}-1}$, and $C=g \sqrt{V^{2}-1}$. Substituting the matrix elements in Eq. (6) into Eqs. (3) and (44), the EPR steering $\mathcal{G}^{A \rightarrow D^{\prime}}$ and $\mathcal{G}^{D^{\prime} \rightarrow A}$ can be obtained.

The gain factor in the classical channel is an important parameter in entanglement swapping. Steerabilities between modes $\hat{A}$ and $\hat{D}^{\prime}$ also depend on the gains in the classical channels. The optimal gains can be obtained by maximizing the steerabilities $\mathcal{G}^{A \rightarrow D^{\prime}}$ and $\mathcal{G}^{D^{\prime} \rightarrow A}$, which are given by

$$
\begin{aligned}
g_{\mathrm{opt}}^{A \rightarrow D^{\prime}} & =\frac{V \sqrt{V^{2}-1}}{V^{2}+1}, \\
g_{\mathrm{opt}}^{D^{\prime} \rightarrow A} & =\frac{V}{\sqrt{V^{2}-1}} .
\end{aligned}
$$

respectively. From the expression of $g_{\mathrm{opt}}^{A \rightarrow D^{\prime}}$ and $g_{\mathrm{opt}}^{D^{\prime} \rightarrow A}$, we see that $g_{\mathrm{opt}}^{A \rightarrow D^{\prime}}$ and $g_{\mathrm{opt}}^{D^{\prime} \rightarrow A}$ are smaller and larger than 1 for any $V$, respectively. They gradually approach 1 from opposite directions as $V$ increases. In the limit of $V \rightarrow \infty$, which corresponds to perfect EPR entanglement, $g_{\mathrm{opt}}^{A \rightarrow D^{\prime}}$ and $g_{\mathrm{opt}}^{D^{\prime} \rightarrow A}$ are both equal to 1 . The difference between Eqs. (7) and (8) comes from the asymmetric property of the output state, i.e., the submatrixes $A \mathbf{I}$ and $B \mathbf{I}$ are different in Eq. (6) even for the ideal case. In the ideal case, i.e., with unit transmission efficiency, detection efficiency, and unit gain in the classical channel, we have $B=3 V-2 \sqrt{V^{2}-1}$ in Eq. (6). It is obvious that $B$ is equal to $A$ only when $V$ is infinite, which corresponds to an infinite squeezing level. However, an infinite squeezing level is impossible because infinite pumping power is required. In realistic cases, modes $\hat{B}$ or/and $\hat{C}$ suffer from transmission losses, so $B$ and $A$ are not equal even if perfect detection efficiency and unit gain are taken.

Loss and noise in quantum channels can lead to decoherence of the quantum state. Especially, excess noise in the quantum channel, which is the noise above the vacuum noise, can lead to the disappearance of squeezing and the sudden death of entanglement [32, 33]. Here, we consider the proposed swapping schemes for Gaussian EPR steering in lossy and noisy channels. The losses in the quantum channels are modeled by beam splitters with transmission efficiencies $T_{1}$ and $T_{2}$, respectively. The excess noise is modeled by environmental thermal states with noise $W_{1}$ and $W_{2}$, respectively. $W_{i}=0(i=1,2)$ means that there is no excess noise and only loss exists in the quantum channel. An optical mode $\hat{o}$ turns into $\sqrt{T} \hat{o}+\sqrt{1-T}(\hat{\nu}+\hat{w})$ after it is transmitted through a lossy and noisy channel, where $\hat{\nu}$ and $\hat{w}$ represent the vacuum state and thermal state, respectively. The detection efficiency $\eta$ of the homodyne detector for modes $\hat{E}$ and $\hat{F}$ is also taken into account, which is modeled by a beam splitter with a transmission efficiency $\eta$. Taking all imperfections into account, the covariance matrix elements in Eq. (6) are $A=V, B=V-2 g \sqrt{\eta T_{2}} \sqrt{V^{2}-1}+$ $g^{2}\left\{2+\eta\left[\left(T_{1}+T_{2}\right)(V-1)-T_{1} W_{1}-T_{2} W_{2}+W_{1}+W_{2}\right]\right\}$, and $C=g \sqrt{\eta T_{1}} \sqrt{V^{2}-1}$, respectively. The corresponding optimal gain $g_{\mathrm{opt}}^{A \rightarrow D^{\prime}}$ in the classical channels can be expressed analytically as 


$$
g_{\mathrm{opt}}^{A \rightarrow D^{\prime}}=\frac{V \sqrt{\eta T_{2}} \sqrt{V^{2}-1}}{2 V+\eta\left\{V\left[\left(T_{1}+T_{2}\right)(V-1)-T_{1} W_{1}-T_{2} W_{2}+W_{1}+W_{2}\right]-\left(V^{2}-1\right) T_{1}\right\}} .
$$
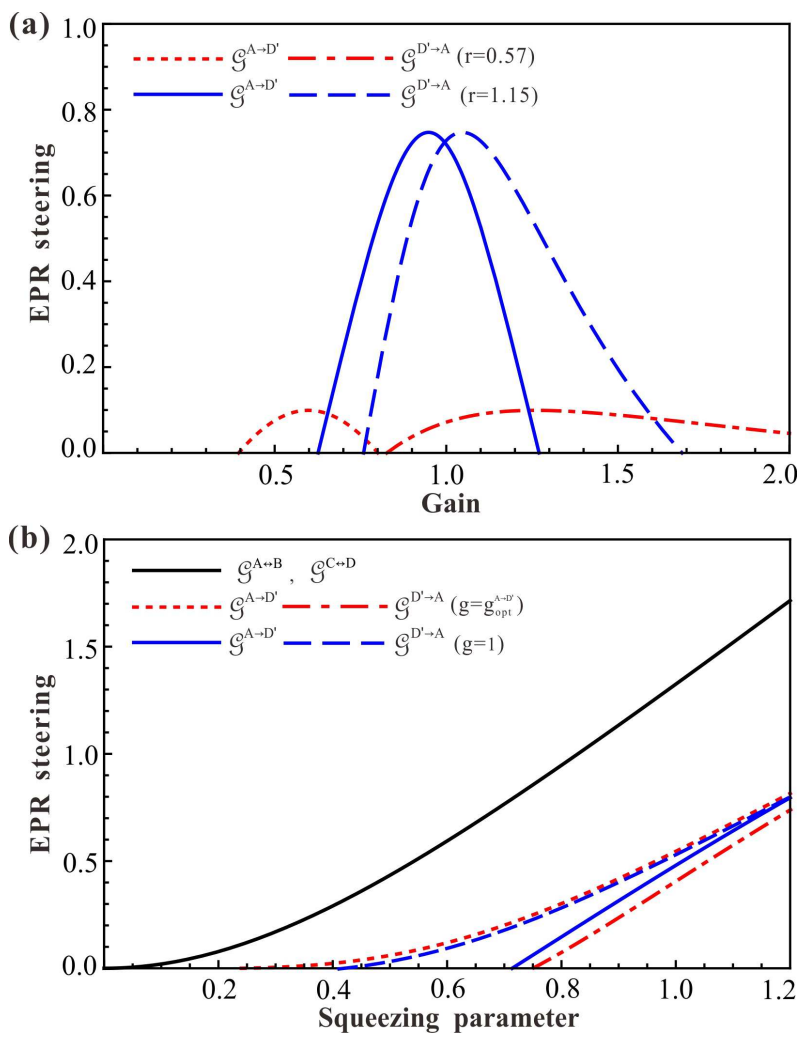

FIG. 2: (a) Dependence of EPR steerability on gain factors in classical channels with two different squeezing parameters. (b) Dependence of steerability on squeezing parameter $r$. The red dotted and dashed-dotted curves represent the steering between modes $\hat{A}$ and $\hat{D}^{\prime}$ when the optimal gain factor $g_{\mathrm{opt}}^{A \rightarrow D^{\prime}}$ is chosen. The blue solid and dashed curves correspond to the steering between $\hat{A}$ and $\hat{D}^{\prime}$ where the unit gain factor is chosen. The black curve shows the steerability of the original EPR resource for comparison.

\section{RESULTS AND DISCUSSIONS}

Figure 2(a) shows the dependence of output EPR steering on gain factors in the classical channels with two different squeezing parameters. The transmission efficiencies $T_{1}$ and $T_{2}$ are chosen as 1 , which means that the swapping process is implemented locally, and the detection efficiency $\eta$ is chosen as $95 \%$. Different from entanglement swapping, the optimal gain factors for $\mathcal{G}^{A \rightarrow D^{\prime}}$ and $\mathcal{G}^{D^{\prime} \rightarrow A}$ are different, which is due to the asymmetry of modes $\hat{A}$ and $\hat{D}^{\prime}$. As $r$ increases, the optimal gain factors for $\mathcal{G}^{A \rightarrow D^{\prime}}$ and $\mathcal{G}^{D^{\prime} \rightarrow A}$ tend to 1 , and the maximum (a)

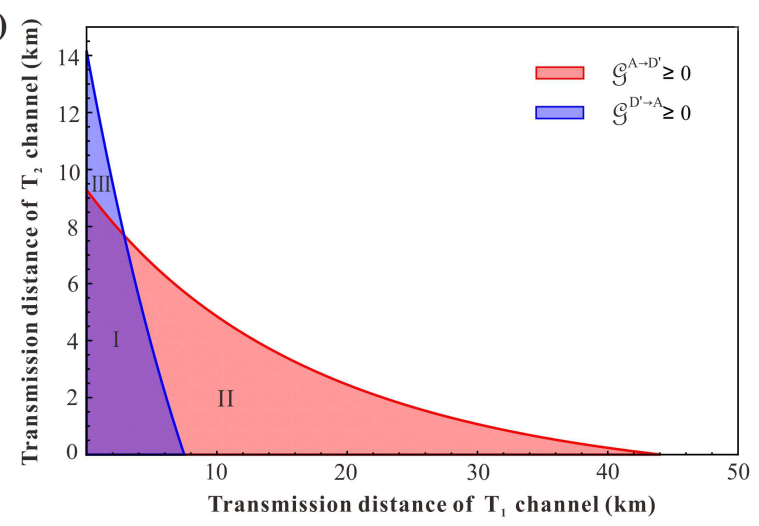

(b)

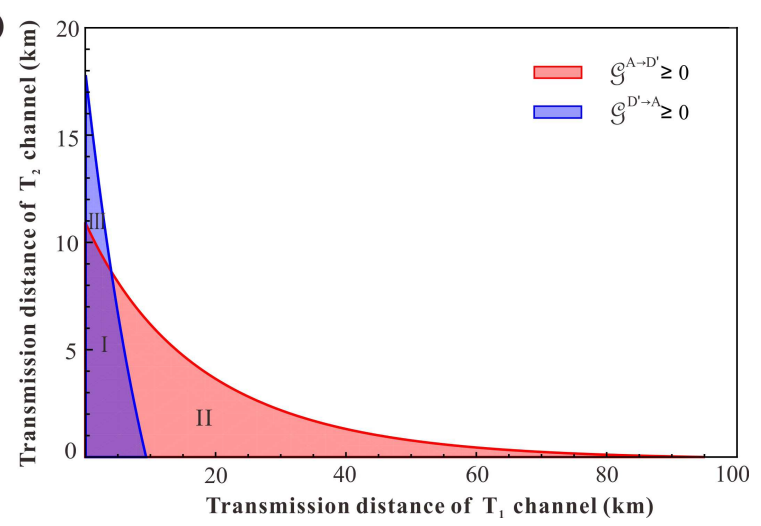

FIG. 3: The transmission distance regions for output EPR steering in lossy channels when the optimal gain factor $g_{\mathrm{opt}}^{A \rightarrow D^{\prime}}$ is chosen. (a) and (b) correspond to a detection efficiency of $95 \%$ and $99.5 \%$, respectively. Regions I, II, and III correspond to two-way EPR steering, and one-way EPR steering for $\mathcal{G}^{A \rightarrow D^{\prime}}$ and $\mathcal{G}^{D^{\prime} \rightarrow A}$, respectively.

steerabilities also substantially increase. Thus $g=1$ corresponds to the ideal swapping operation in the limit of infinite squeezing.

Figure 2(b) shows the dependence of output EPR steering on the squeezing parameter $r$ with optimal gain $g_{\mathrm{opt}}^{A \rightarrow D^{\prime}}$ and unit gain, respectively, where the transmission efficiencies $T_{1}$ and $T_{2}$ are chosen as 1 and the detection efficiency $\eta$ is chosen as $95 \%$. The black curve shows the steerability of the original EPR resource for comparison. The steerability of modes $\hat{A}$ and $\hat{D}^{\prime}$ is lower than that of the original EPR entangled states $(\hat{A}, \hat{B})$ and $(\hat{C}, \hat{D})$. When the unit gain is chosen, EPR steering $\mathcal{G}^{A \rightarrow D^{\prime}}$ and $\mathcal{G}^{D^{\prime} \rightarrow A}$ exist only when the squeezing parameter $r$ is larger than 0.72 and 0.42 , respectively (blue 

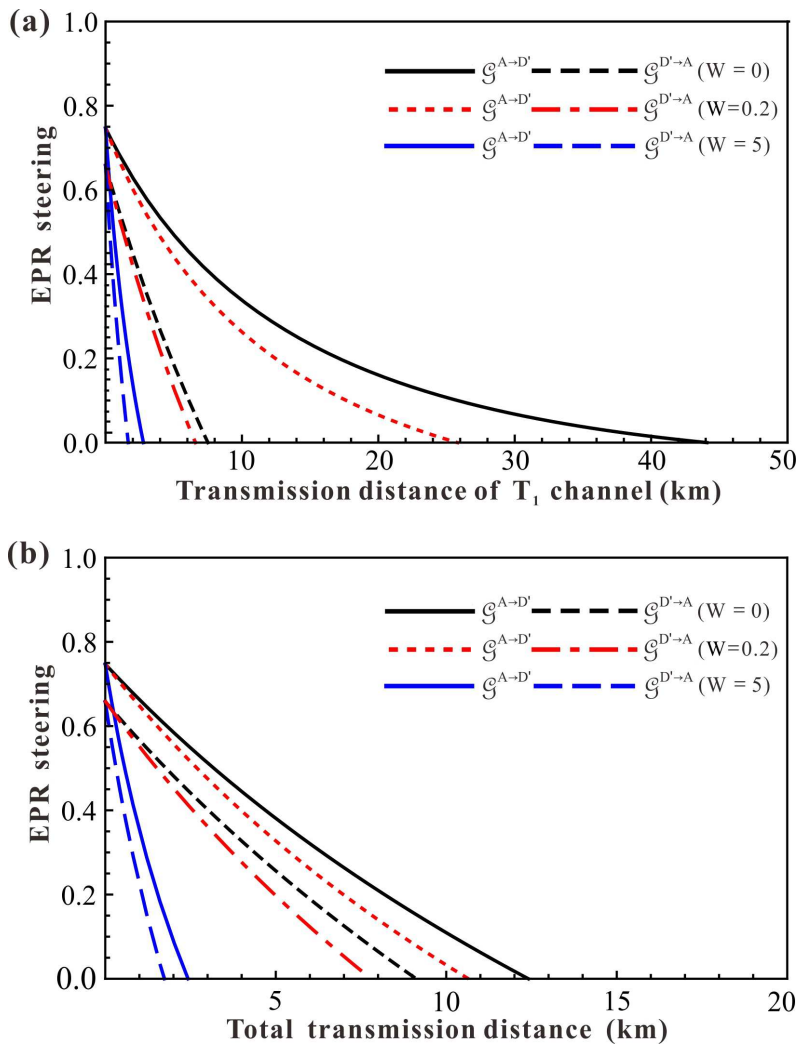

FIG. 4: Dependence of EPR steering on the transmission distance with different excess noise for (a) the single-channel scheme and (b) the symmetric dual-channel scheme. The excess noise is taken as $\mathrm{W}=0,0.2$, and 5 for comparison.

solid and dashed curves). One-way steering $\mathcal{G}^{D^{\prime} \rightarrow A}$ is observed in the range $0.42<r<0.72$. When the optimal gain $g_{\mathrm{opt}}^{A \rightarrow D^{\prime}}$ is chosen, EPR steering $\mathcal{G}^{A \rightarrow D^{\prime}}$ and $\mathcal{G}^{D^{\prime} \rightarrow A}$ can be obtained when the squeezing parameter is larger than 0.24 and 0.75 , respectively (red dotted and dasheddotted curves). One-way steering $\mathcal{G}^{A \rightarrow D^{\prime}}$ is observed in the range $0.24<r<0.75$. As the squeezing parameter $r$ increases, these four curves tend to overlap each other. Please note that although the optical mode is not transmitted over a lossy channel in this case, one-way EPR steering is also presented. This is because the symmetry of the output state is broken after the swapping process, just as the previous observed one-way EPR steering in a lossy channel [12].

Comparing the EPR steering $\mathcal{G}^{A \rightarrow D^{\prime}}$ with unit gain and optimal gain $g_{\mathrm{opt}}^{A \rightarrow D^{\prime}}$, the required squeezing parameter for $\mathcal{G}^{A \rightarrow D^{\prime}}$ is reduced from 0.72 to 0.24 with optimal gain $g_{\mathrm{opt}}^{A \rightarrow D^{\prime}}$. Comparing the EPR steering $\mathcal{G}^{D^{\prime} \rightarrow A}$ with unit gain and optimal gain $g_{\mathrm{opt}}^{A \rightarrow D^{\prime}}$, the required squeezing parameter for $\mathcal{G}^{D^{\prime} \rightarrow A}$ is increased from 0.42 to 0.75 by choosing the optimal gain $g_{\mathrm{opt}}^{A \rightarrow D^{\prime}}$. This is because the optimal gain $g_{\mathrm{opt}}^{A \rightarrow D^{\prime}}$ is the maximization of steerability $\mathcal{G}^{A \rightarrow D^{\prime}}$. If we choose the optimal gain $g_{\mathrm{opt}}^{D^{\prime} \rightarrow A}$, the re- quired squeezing parameter for $\mathcal{G}^{D^{\prime} \rightarrow A}$ will be reduced while that for $\mathcal{G}^{A \rightarrow D^{\prime}}$ will be increased. The physical reason for this phenomenon comes from the asymmetric property of EPR steering. Here, we choose the optimal gain factor $g_{\mathrm{opt}}^{A \rightarrow D^{\prime}}$ as an example to present the results.

Figure 3 shows the transmission distance regions for EPR steering in lossy but noiseless quantum channels, where the gain factor in the classical channel is taken as $g_{\mathrm{opt}}^{A \rightarrow D^{\prime}}$. Here, we consider the transmission loss $\alpha=0.2$ $\mathrm{dB} / \mathrm{km}$ in the telecommunication fiber and the squeezing parameter $r=1.15$ (corresponding to $10 \mathrm{~dB}$ squeezing). The detection efficiency is chosen as $95 \%$ and $99.5 \%$ in Figs. 3(a) and 3(b), respectively. In the single-channel scheme [see the transmission distance of the $T_{1}$ channel in Fig. 3(a)], the maximum transmission distances for EPR steering $\mathcal{G}^{A \rightarrow D^{\prime}}$ and $\mathcal{G}^{D^{\prime} \rightarrow A}$ are 45 and $7.6 \mathrm{~km}$, respectively. When the detection efficiency is improved to $99.5 \%$, the transmission distances for EPR steering $\mathcal{G}^{A \rightarrow D^{\prime}}$ and $\mathcal{G}^{D^{\prime} \rightarrow A}$ in the single-channel scheme can be increased to 95 and $9.5 \mathrm{~km}$, respectively [Fig. 3(b)]. The longer transmission distance for $\mathcal{G}^{A \rightarrow D^{\prime}}$ is obtained because the gain factor is taken as $g_{\mathrm{opt}}^{A \rightarrow D^{\prime}}$. We also see that the transmission distance of the single-channel scheme is much longer than that of the symmetric dual-channel scheme.

With the increase of transmission distance in the quantum channels, two-way EPR steering (region I) can be turned to either one-way EPR steering $\mathcal{G}^{A \rightarrow D^{\prime}}$ (region II) or $\mathcal{G}^{D^{\prime} \rightarrow A}$ (region III), respectively. The direction of one-way EPR steering can be changed at the crossover point of the two boundary curves for $\mathcal{G}^{A \rightarrow D^{\prime}} \geq 0$ and $\mathcal{G}^{D^{\prime} \rightarrow A} \geq 0$ in the dual-channel scheme. As shown in Fig. 3(a), one-way EPR steering $\mathcal{G}^{D^{\prime} \rightarrow A}$ can be observed when the transmission distance of mode $\hat{B}$ is shorter than 2.9 $\mathrm{km}$ (region III), while the one-way EPR steering $\mathcal{G}^{A \rightarrow D^{\prime}}$ can be obtained when the transmission distance of mode $\hat{B}$ is longer than $2.9 \mathrm{~km}$ (region II).

Here, we explain the physical reason for the change of the EPR steering direction. The EPR steering regions I, II, and III in Fig. 3 correspond to the results of $\mathcal{G}^{A \rightarrow D^{\prime}}>0$ and $\mathcal{G}^{D^{\prime} \rightarrow A}>0, \mathcal{G}^{A \rightarrow D^{\prime}}>0>\mathcal{G}^{D^{\prime} \rightarrow A}$, and $\mathcal{G}^{D^{\prime} \rightarrow A}>0>\mathcal{G}^{A \rightarrow D^{\prime}}$, respectively. From the expression of steerabilities in Eqs. (3) and (4), it can be clearly seen that the conditions corresponding to the EPR steering regions I, II, and III in Fig. 3 are $\operatorname{det} \sigma_{A}$ and $\operatorname{det} \sigma_{D^{\prime}}>\operatorname{det} \sigma_{A D^{\prime}}, \operatorname{det} \sigma_{A}>\operatorname{det} \sigma_{A D^{\prime}}>\operatorname{det} \sigma_{D^{\prime}}$, and $\operatorname{det} \sigma_{D^{\prime}}>\operatorname{det} \sigma_{A D^{\prime}}>\operatorname{det} \sigma_{A}$, respectively. Two-way EPR steering can be transformed to one-way EPR steering $A \rightarrow D^{\prime}\left(D^{\prime} \rightarrow A\right)$ if the asymmetry of the state exceeds the boundary $\operatorname{det} \sigma_{A D^{\prime}}=\operatorname{det} \sigma_{D^{\prime}}\left(\operatorname{det} \sigma_{A D^{\prime}}=\right.$ $\operatorname{det} \sigma_{A}$ ) between regions I and II (I and III). However, it must be pointed out that the asymmetric property of the two-mode quantum state is only a necessary condition for one-way EPR steering. In other words, a two-mode quantum state exhibiting one-way EPR steering must be an asymmetric state, while a two-mode quantum state exhibiting two-way EPR steering may also be an asym- 
metric state.

The dependence of EPR steering on the transmission distance in noisy channels is shown in Fig. 4. Figures 4(a) and 4(b) show the single-channel scheme and symmetric dual-channel scheme, respectively. The squeezing parameter $r=1.15$ is chosen, and the excess noise $W$ is taken as $0,0.2$, and 5 (in units of shot-noise level), respectively. The optimal gain factor $g_{\mathrm{opt}}^{A \rightarrow D^{\prime}}$ in the classical channel is chosen, thus the steerability $\mathcal{G}^{A \rightarrow D^{\prime}}$ is always larger than $\mathcal{G}^{D^{\prime} \rightarrow A}$ at each noise level. For simplification, the distances and noise in the two quantum channels are chosen to be equal in the dual-channel scheme. It is obvious that the transmission distance in the single-channel scheme is much longer than that of the symmetric dualchannel scheme at the same excess noise level. The transmission distances decrease dramatically as excess noise increases in both schemes. The sudden death of EPR steering can occur when there is larger excess noise in the quantum channel.

\section{CONCLUSION}

Comparing with the Gaussian entanglement swapping scheme 20 23], the same procedure is used in the presented swapping schemes of Gaussian EPR steering. There are two differences between the swapping of Gaussian EPR steering and Gaussian entanglement. First, in the swapping of Gaussian EPR steering, the obtained steerabilities of the two remote modes are asymmetric, while the obtained entanglement of the two remote modes are the same in Gaussian entanglement swapping. Second, the dependence on the squeezing parameter is different when the optimal gain is chosen. When the optimal gain in the classical channel is chosen, higher squeezing is required to complete the swapping of Gaussian EPR steering, while Gaussian entanglement swapping can be completed with nonzero squeezing [23].

In conclusion, two swapping schemes of Gaussian EPR steering, a single-channel scheme and dual-channel scheme, are presented. EPR steering is observed between two independent quantum modes without a direct interaction by using the technique of entanglement swapping. The transmission distances of the single-channel scheme and dual-channel scheme are compared, and the maximum transmission distance can be obtained by using the single-channel scheme. The transmission distances are limited by the squeezing of the Gaussian EPR state and the detection efficiency of the homodyne detector in the joint measurement. If an EPR state with higher squeezing and a homodyne detector with higher detection efficiency are used, a longer transmission distance can be obtained.

One-way EPR steering is presented after the swapping, which is an inherent property of EPR steering. The change in the EPR steering direction is observed in the dual-channel scheme, which is related to the asymmetric property of the output state. In noisy quantum channels, the transmission distance decreases dramatically with an increase of excess noise in quantum channels. The presented schemes can be applied in quantum communication networks with EPR steering.

\section{ACKNOWLEDGMENT}

This research was supported by the NSFC (Grant No. 61475092, No. 11522433, and No. 61601270), the program of Youth Sanjin Scholar, the Applied Basic Research Program of Shanxi province (Grant No. 201601D202006), and National Basic Research Program of China (Grant No. 2016YFA0301402).
[1] A. Einstein, B. Podolsky, and N. Rosen, Phys. Rev. 47, 777 (1935).

[2] E. Schrödinger, Proc. Cambridge Philos. Soc. 31, 555 (1935).

[3] E. Schrödinger, Proc. Cambridge Philos. Soc. 32, 446 (1936).

[4] J. S. Bell, Physics 1, 195 (1964).

[5] R. Horodecki, P. Horodecki, M. Horodecki, and K. Horodecki, Rev. Mod. Phys. 81, 865 (2009).

[6] H. M. Wiseman, S. J. Jones, and A. C. Doherty, Phys. Rev. Lett. 98, 140402 (2007).

[7] S. J. Jones, H. M. Wiseman, and A. C. Doherty, Phys. Rev. A 76, 052116 (2007).

[8] P. Skrzypczyk, M. Navascués, and D. Cavalcanti, Phys. Rev. Lett. 112, 180404 (2014).

[9] C. Branciard, E. G. Cavalcanti, S. P. Walborn, V. Scarani, and H. M. Wiseman, Phys. Rev. A 85, 010301 (2012).

[10] M. Piani and J. Watrous, Phys. Rev. Lett. 114, 060404 (2015).
[11] Q. He, L. Rosales-Zárate, G. Adesso, and M. D. Reid, Phys. Rev. Lett. 115, 180502 (2015).

[12] V. Händchen, T. Eberle, S. Steinlechner, A. Samblowski, T. Franz, R. F. Werner, and R. Schnabel, Nat. Photonics 6, 596 (2012).

[13] S. Armstrong, M. Wang, R. Y. Teh, Q. H. Gong, Q. Y. He, J. Janousek, H. A. Bachor, M. D. Reid, and P. K. Lam, Nat. Phys. 11, 167 (2015).

[14] J. Bowles, T. Vértesi, M. T. Quintino, and N. Brunner, Phys. Rev. Lett. 112, 200402 (2014).

[15] M. T. Quintino, T. Vértesi, D. Cavalcanti, R. Augusiak, M. Demianowicz, A. Acín, and N. Brunner, Phys. Rev. A 92, 032107 (2015).

[16] D. A. Evans and H. M. Wiseman, Phys. Rev. A 90, 012114 (2014).

[17] S. Wollmann, N. Walk, A. J. Bennet, H. M. Wiseman, and G. J. Pryde, Phys. Rev. Lett. 116, 160403 (2016).

[18] K. Sun, X.-J. Ye, J.-S. Xu, X.-Y. Xu, J.-S. Tang, Y.-C. Wu, J.-L. Chen, C.-F. Li, and G.-C. Guo, Phys. Rev. Lett. 116, 160404 (2016). 
[19] M. Zukowski, A. Zeilinger, M. A. Horne, and A. K. Ekert, Phys. Rev. Lett. 71, 4287 (1993).

[20] R. E. S. Polkinghorne and T. C. Ralph, Phys. Rev. Lett. 83, 2095 (1999).

[21] S. M. Tan, Phys. Rev. A 60, 2752 (1999).

[22] P. van Loock and S. L. Braunstein, Phys. Rev. A 61, 010302(R) (1999).

[23] J. Zhang, C. Xie, and K. Peng, Phys. Lett. A 299, 427 (2002).

[24] L.-M. Duan, M. D. Lukin, J. I. Cirac, and P. Zoller, Nature (London) 414, 413 (2001).

[25] J.-W. Pan, D. Bouwmeester, H. Weinfurter, and A. Zeilinger, Phys. Rev. Lett. 80, 3891 (1998).

[26] X. Jia, X. Su, Q. Pan, J. Gao, C. Xie, and K. Peng, Phys.
Rev. Lett. 93, 250503 (2004).

[27] S. Takeda, M. Fuwa, P. van Loock, and A. Furusawa, Phys. Rev. Lett. 114, 100501 (2015).

[28] C.-Y. Lu, T. Yang, and J.-W. Pan, Phys. Rev. Lett. 103, 020501 (2009).

[29] L. Ma, and X. Su, Opt. Express 22, 015894 (2014).

[30] X. Su, C. Tian, X. Deng, Q. Li, C. Xie and K. Peng, Phys. Rev. Lett. 117, 240503 (2016).

[31] I. Kogias, A. R. Lee, S. Ragy, and G. Adesso, Phys. Rev. Lett. 114, 060403 (2015).

[32] X. Deng, S. Hao, C. Tian, X. Su, C. Xie and K. Peng, Appl. Phys. Lett. 108, 081105 (2016).

[33] X.-L. Su, Chin. Phys. B, 22, 080304 (2013). 